\section{La spironolactone: somnifère du VIH dans les lymphocytes T ?}

Benoît Lacombe, Bertha Cecilia Ramírez
Équipe rétrovirus, quiescence et prolifération. Institut Cochin, Inserm U1016, CNRS, UMR8104, université Paris Descartes, Sorbonne Paris Cité, Paris, France. cecilia.ramirez@inserm.fr

(cyclin dependent kinase 7), ce qui permet d'initier la transcription.

Des molécules actuellement en cours d'évaluation, comme le triptolide (TPL) [2] et le didéhydro-cortistatin A ( $\mathrm{dCA})$ [3], montrent des résultats prometteurs quant à l'inhibition de la transcription. Le TPL interagit avec la protéine Tat et inhibe son activité. II a également des effets sur d'autres composants de la machinerie de transcription, en particulier sur l'ARN Pol II [4] et la protéine XPB [5]. La molécule dCA, quant à elle, se lie de manière covalente à la protéine Tat empêchant son action lors de la transcription du génome viral [3].

Nous avons entrepris de caractériser le rôle de la protéine XPB au cours du cycle du VIH-1, suite à la publication d'observations contradictoires. En effet, XPB a été décrite par certains auteurs comme un facteur antiviral $[6,7]$ alors que, pour d'autres, elle semble être nécessaire à la réplication du virus $[8,9]$. Nous avons étudié l'activité de XPB dans les lymphocytes $T$ en profitant de l'effet de la spironolactone (SP) sur cette protéine. La SP est une molécule utilisée en clinique quotidiennement depuis plusieurs décennies comme antagoniste de l'aldostérone dans le traitement de l'hypertension. En 2014, Alekseev et ses collaborateurs ont démontré que la molécule SP dégrade rapidement XPB, de manière spécifique et réversible [10]. En 2017, les mêmes auteurs ont utilisé la SP pour redéfinir le rôle de la protéine XPB au cours de la transcription [11]. Ils ont alors proposé un nouveau modèle d'initiation de la transcription, dans lequel la dégradation de 


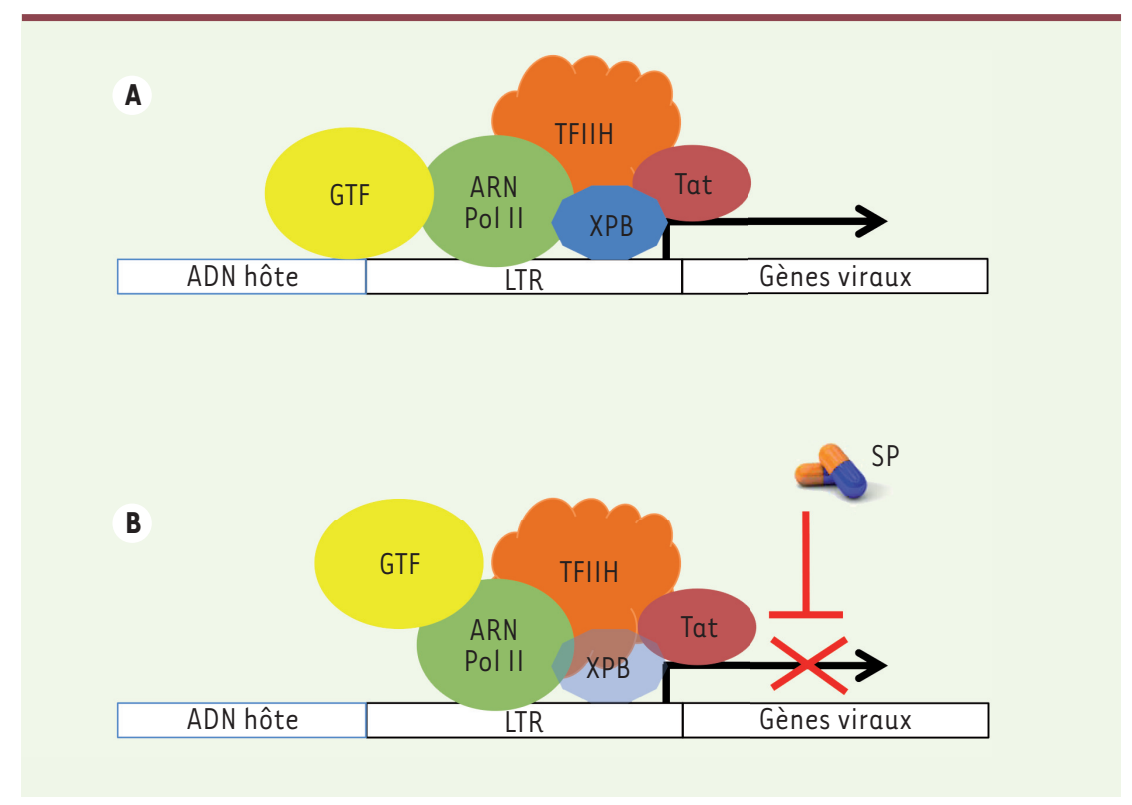

Figure 1. Effet de la spironolactone (SP) sur la transcription de la séquence LTR du virus de l'immunodéficience humaine (VIH). A. Après intégration, la séquence LTR (long terminal repeat) peut être transcrite grâce au recrutement des facteurs généraux de transcription (GTF), de l'ARN Polymérase II (Pol II) ainsi que du complexe de transcription TFIIH constitué, entre autres, de la protéine Xeroderma pigmentosum group $B(\mathrm{XPB})$. La protéine virale Tat favorise la transition et la progression de la transcription de l'initiation à l'élongation. $B$. En présence de spironolactone $(S P)$, la protéine XPB est dégradée et la transcription virale dépendante de Tat est bloquée. TFIIH : complexe de transcription II humain ; Tat : trans activator of transcription.

la protéine XPB par la SP n'affecte pas l'activité transcriptionnelle du complexe TFIIH, mettant ainsi en évidence un mécanisme d'initiation possible en l'absence de XPB. Selon Alekseev et ses collaborateurs, cette protéine agirait comme un inhibiteur de l'initiation de la transcription. La SP outrepasserait cette inhibition pour favoriser un mécanisme d'initiation de la transcription indépendant de l'ATP [11].

Nous avons publié récemment, dans la revue Journal of Virology, les résultats surprenants obtenus dans notre étude sur la SP [12]. En effet, celle-ci bloque très fortement la multiplication du VIH dans les lymphocytes $T$, sans affecter les processus moléculaires fondamentaux dans lesquels le complexe TFIIH est impliqué. Dans les cellules de la lignée lymphoïde T Jurkat en culture, mais également dans des lymphocytes T CD4 ${ }^{+}$ primaires isolés du sang de donneurs sains, la SP induit une dégradation de laires évalués dans des lymphocytes $T$ en culture. L'ensemble des résultats que nous avons recueillis, montre que dans les lymphocytes $T C D 4^{+}, X P B$ est un facteur nécessaire à la réplication du virus VIH et que la SP n'est pas seulement cellules en culture, mais qu'elle bloque également la production virale dans des cellules qui sont infectées depuis pluefficace au début de l'infection des sieurs jours. Différentes expériences ont permis de montrer que cette inhibition de l'infection du VIH par la SP dépendait de la présence de la protéine Tat. En effet, en l'absence de Tat, la SP n'a pas d'effet sur la transcription basale du promoteur LTR du VIH, ou sur la transcription du virus, après activation par le TNF- $\alpha$ (tumor necrosis factor $\alpha$ ) ou le PMA (phorbol 12-myristate 13-acétate $)^{1}$. En revanche, et de façon surprenante, la SP bloque la transcription du génome viral qui dépend de Tat. Cet effet bloquant est spécifique du promoteur du VIH : Ia SP n'a pas d'effet sur la transcription du promoteur d'un autre virus tel que le cytomégalovirus (CMV) [12]. Notre étude a donc mis en évidence l'effet antiviral de la SP sur le VIH (Figure 2). Elle démontre également que la protéine XPB est un facteur nécessaire pour l'infection des lymphocytes T par celui-ci.

Nos travaux actuels examinent la capacité de la SP à bloquer la réactivation de la transcription de virus latents. Cette activité de la SP pourrait permettre de bloquer la réactivation transcriptionnelle des réservoirs viraux chez des patients sous traitement antirétroviral. Ainsi, ce blocage de la transcription, dans les cellules réservoirs, pourrait conduire à la mise en sommeil du virus, tout au long de la vie du patient. Cette nouvelle piste pour le traitement du VIH ouvre des possibilités dans le développement d'un inhibiteur transcriptionnel spécifique et efficace, dans le cadre d'une cure fonctionnelle du Sida. De nombreuses recherches sont cependant encore nécessaires afin de confirmer l'inhibition de la réactivation de la transcription du VIH dans des cellules de patients infectées par le virus.

L'action de la SP sur la multiplication du VIH est innovante : elle cible un facteur cellulaire et non plus viral comme les autres antirétroviraux ou molécules testées actuellement. Cibler un facteur

\footnotetext{
${ }^{1}$ Qui est un inducteur de différenciation et agit comme stimulus de la réplication virale.
} 


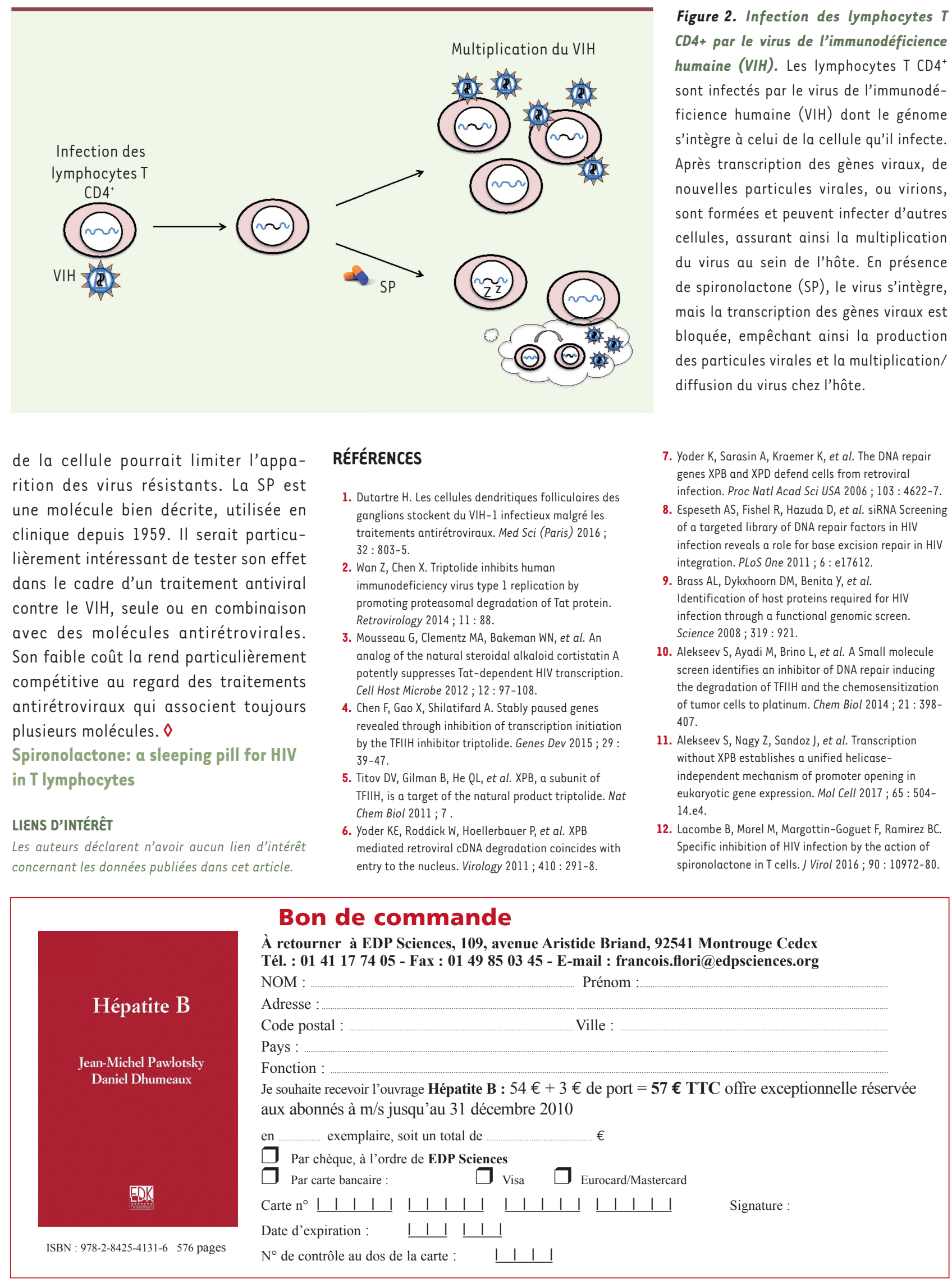

